\title{
TOWARDS TRANSCRIPTOMICS AS A PRIMARY TOOL FOR RARE DISEASE INVESTIGATION
}

Montgomery, S.B. ${ }^{1}$, Bernstein, J.A ${ }^{2}$., Wheeler, M.T. ${ }^{3}$

${ }^{1}$ Departments of Genetics and Pathology, Stanford University, CA, USA

${ }^{2}$ Department of Pediatrics, Stanford University School of Medicine, Stanford, CA, USA

${ }^{3}$ Division of Cardiovascular Medicine, Department of Medicine, Stanford University, CA, USA

\begin{abstract}
In the past five years transcriptome or RNA-sequencing (RNA-seq) has steadily emerged as a complementary assay for rare disease diagnosis and discovery. In this perspective, we summarize several recent developments and challenges in use of RNA-seq for rare disease investigation. Using an accessible patient sample, such as blood, skin, or muscle, RNA-seq enables the assay of expressed RNA transcripts. Analysis of RNA-seq allows the identification of aberrant or outlier gene expression and alternative splicing as functional evidence to support rare disease study and diagnosis. Further, many types of variant effects can be profiled beyond coding variants, as the consequences of non-coding variants that impact gene expression and splicing can be directly observed. This is particularly apparent for structural variants which disproportionately underlie outlier gene expression and for splicing variants where RNA-seq can both measure aberrant canonical splicing and detect deep intronic effects. However, a major potential limitation of RNA-seq in rare disease investigation is the developmental and cell typespecificity of gene expression as a pathogenic variant's effect may be limited to a specific spatiotemporal context and access to a patient's tissue sample from the relevant tissue and timing of disease expression may not be possible. We speculate that as advances in computational methods and emerging experimental techniques overcome both developmental and cell type-specificity, there will be broadening use of RNA sequencing and multi-omics in rare disease diagnosis and delivery of precision health.
\end{abstract}

\section{MEASURING RARE VARIANT EFFECTS USING TRANSCRIPTOMICS}

Transcriptome or RNA-sequencing in specific cell types or tissues can provide robust quantification for the expression levels of more than 8000 genes and further tens of thousands of splice junctions, providing extensive coverage of a broad range of molecular events. In contrast to targeted methods of RNA quantification such as RT-PCR, this approach can provide a broad view of transcription-related molecular events. In addition, the types of molecular events evaluated by RNA-seq extend beyond expression and splicing levels of known gene products as identification and quantification of non-coding genes, novel transcripts, fusion genes, retained introns, alternative poly-adenylation and transcription starts can be determined. Further, additional molecular signals are also quantifiable including allele-specific expression, nonsense-mediated decay and RNA-editing.

Each transcript measured by RNA-seq is subsequently amenable to genetic analyses. When profiled in human population samples, genetic association analyses have uncovered abundant quantitative trait loci (QTL) for common genetic variants (The GTEx Consortium 2020). However, extending these analyses to rare variant effects has been more challenging due to increased uncertainty around effect sizes and the abundance of rare variants to test in any human population sample (Keinan and Clark 2012; Bomba, Walter, and Soranzo 2017). To 
mitigate some of these challenges and focus on impactful rare variants, analyses can be restricted to rare variants associated with outlier expression measurements. As such, we and others have shown that genes with outlier expression in an individual are enriched in gene proximal rare variants ( Li et al. 2017; Zhao et al. 2016; Montgomery et al. 2011; Zeng et al. 2015). These enrichments were further significant for all major classes of genetic variants but were most striking for rare structural variants reinforcing the ongoing needs for accurately identifying structural variants in individual genomes and the use of transcriptomes to guide interpreting variant effects (Ferraro et al. 2020). Furthermore, these enrichments informed both protein-coding and non-coding variant effects; rare, protein-truncating variants were highly enriched in individuals with outlier gene expression due to the effects of nonsense-mediated decay on gene expression and, rare, predicted splicing variants were highly enriched in individuals with outlier splicing levels. To continue the discovery of outlier-associated rare variants will require that future transcriptome studies are complemented by high quality whole genome data. Further, as these studies have focused predominantly on expression and splicing outliers, other outlier molecular effects from the range of multi-omics assays are only beginning to be systematically integrated with transcriptome data (Kopajtich et al. 2021).

When considering the potential for transcriptome sequencing for rare disease diagnosis, these studies in healthy individuals have demonstrated that outlier effects can be driven by diverse categories of rare variants. They have demonstrated that RNA-seq can facilitate detecting the effects of specific candidate variants, particularly for splicing. Further, they have demonstrated that computational methods that combine both whole genome and RNA-seq data from the same individual can enhance the prioritization of large-effect, rare variants and such variants can have increased impacts on diverse traits and diseases ( $\mathrm{Li}$ et al. 2017; Ferraro et al. 2020). However, there are few other computational methods that integrate individual genomes and transcriptomes to prioritize rare variants, particularly in comparison to variant effect prediction tools that use genome data alone. This is an area of method development that will grow as future approaches combine advances in machine learning for variant effect prediction with individual -omics data to improve discovery of pathogenic rare variants.

\section{DIAGNOSTIC YIELD OF TRANSCRIPTOMICS IN RARE DISEASES.}

It is estimated that the current diagnostic yield of DNA sequencing is $25-30 \%$ in large, heterogeneous rare disease cohorts (Clark et al. 2018; Yang et al. 2013; Jacob et al. 2013; Lee et al. 2014; Yang et al. 2014; Iglesias et al. 2014; Posey et al. 2016; Deciphering Developmental Disorders Study 2017; Tan et al. 2017). In homogeneous rare diseases cohorts, the diagnostic yield can range from 40-60\% (Wortmann et al. 2015; Ankala et al. 2015; Shashi et al. 2014; Splinter et al. 2018; Yuen et al. 2015; 100,000 Genomes Project Pilot Investigators et al. 2021). To investigate further increases to this yield, a number of studies in the past 5 years have used RNA sequencing (Cummings et al. 2017; Kremer et al. 2017; Frésard et al. 2019; Lee et al. 2020; Murdock et al. 2021; Gonorazky et al. 2019; Rentas et al. 2020; Yépez et al. 2021). The public availability of RNA-seq data from rare disease cohorts is poised to accelerate; for example, the Undiagnosed Disease Network has shared RNA-seq data for 816 patient samples (phs001232.v4.p2) and the NIH Centers for Mendelian Genomics Consortium have generated RNA-seq data for 714 patient samples (Baxter et al. 2022). Across these research studies, the use of transcriptomics has predominantly been a secondary activity aimed to evaluate if 
aberrant expression or splicing events can improve identification of pathogenic variants in patients where DNA sequencing alone has not yielded a definitive genetic cause. Despite recent advances, clinical transcriptomic testing for Mendelian conditions is currently available from only a few reference laboratories (https://www.ncbi.nlm.nih.gov/gtr/).

The earliest application of transcriptomics to enhance diagnostic yield in a DNAsequenced rare disease cohort was conducted by Cummings et al. to survey 50 patients with genetically undiagnosed rare muscle disorders (Cummings et al. 2017). Cummings et al. focused on analysis of muscle tissue in comparison to 180 healthy controls muscle samples from the NIH Common Fund Genotype Tissue Expression (GTEx; gtexportal.org) project to identify predominantly splice-altering variants and reported a diagnostic yield of $35 \%$. Kremer et al., conducted another study where they generated fibroblast transcriptomes for 48 patients with undiagnosed rare mitochondriopathies and compared them to a cohort of 105 patients (including the original 48) to achieve a diagnostic yield of $10 \%$ (Kremer et al. 2017). Our own work evaluated the utility of blood transcriptomes for diverse rare disease cases encountered by a clinical genomics service (Frésard et al. 2019). We generated blood transcriptomes for a heterogenous rare disease cohort of 94 patients and compared them to nearly 1000 healthy control samples to achieve an incremental diagnostic yield of $7.5 \%$. We further estimated the need for hundreds of healthy individual samples per tissue type to achieve robust estimates of a patient's outlier effect as increasing enrichments of outliers in loss-of-function intolerant genes were observed as a function of healthy reference sample size. Since these studies, a number of comparable analyses have systematically used RNA-seq to supplement rare disease investigations reporting increases in diagnostic yields ranging from 7-36\% (Lee et al. 2020; Murdock et al. 2021; Rentas et al. 2020; Gonorazky et al. 2019; Yépez et al. 2021) and a growing number of reviews provide additional insights into this area of research (Saeidian et al. 2020; Ergin, Kherad, and Alagoz 2022; Macken et al. 2021; Lord and Baralle 2021)

Despite the new diagnostic opportunities presented by these studies, they have also presented different diagnostic yields and collectively indicated several key factors when using transcriptome sequencing in a rare disease setting. A primary factor is the extent of prior information on both the mode of inheritance and likely causal genes. Relatively easier cases to diagnose using transcriptome sequencing are recessive diseases with known causal gene(s) and a yet-to-be-annotated pathogenic variant. Here, transcriptome sequencing can nominate or rule out splice or regulatory effects within the focus of the causal gene or gene set. This is further facilitated if one of the pathogenic alleles is already identified and the underlying cause is suspected to involve compound heterozygosity with the second, yet-to-be-discovered allele. At least three of the solved cases from Cummings et al. involved compound heterozygosity of a protein-coding and splicing variant. The most challenging cases to supplement with transcriptome sequencing remain somatic mosaic disorders (Ayturk et al. 2016) and disorders where candidate genes remain unknown and few, if any, clinical diagnoses have been made.

Another critical factor to assessing the incremental diagnostic yield of RNA-seq in rare disease cohorts was study design. Unlike genome sequencing (NICUSeq Study Group et al. 2021), there has yet to be a randomized clinical trial on the diagnostic yield for RNAsequencing. As such, existing studies are either exploratory or retrospective and can vary on how they define solved cases. The study design in Cummings et al. focused on rare myopathies using muscle RNA-seq and, ultimately, 15 of their 17 novel diagnoses were within 4 well-known 
myopathy genes: COL6A1, DMD, NEB and TTN. Cases were diagnosed if a complete genetic diagnosis could be inferred in the context of an outlier splice event. Kremer et al.'s study design focused on rare mitochondriopathies using fibroblast RNA-seq. The authors were able to report a novel disease association for TIMMDC1 and considered cases solved if a disease-associated variant was detected and RNA-seq effects were validated in proteomics and/or biochemical assays. Fresard et al.'s study design focused on a heterogeneous mix of rare diseases using blood RNA-seq and was limited to defining a case solved only when clinically curated variants and outlier genes converged, and a complete genetic explanation was possible. For each study, future refinements of candidate gene sets and follow-up validation experiments are expected to increase the diagnostic yield. To this point, both Kremer et al. and Fresard et al. indicated a high number of promising expression and splicing outlier genes where a complete genetic diagnosis was yet to be confirmed.

\section{IMPACTS OF DEVELOPMENTAL AND CELL TYPE-SPECIFICITY.}

An often-discussed challenge with use of transcriptome sequencing to aid rare disease diagnosis is the unknown impact of developmental and cell type-specificity of gene expression. Several genetic diseases can occur due to mutations in tissue restricted transcripts in difficult to access tissues (i.e. cerebral cortex, myocardium). Multiple congenital disorders are already known to have developmental and cell type specific etiologies driven by specific enhancer mutations (Claringbould and Zaugg 2021). Existing transcriptome-based rare disease studies have profiled a range of relatively accessible tissues and cell lines from blood, fibroblasts, lymphoblastoid cell lines or muscle. However, if a disease-causing rare variant's impact is restricted in time and space, profiling a more readily sampled cell type could fail to provide any meaningful extra diagnostic information and add additional patient burden and cost.

Assessing the extent and impact of cell type-specificity of genetic effects was a major rationale for the GTEx project's survey of gene expression and splicing across the human body (The GTEx Consortium 2020). GTEx identified a U-shaped pattern for specificity of common variant effects with gene regulatory effects being either highly shared or highly-tissue restricted. GTEx also identified that a variant's proximity to a gene and a gene's expression level were good indicators of whether an effect would be observed in an unassayed tissue. The observation is expected to extend to rare variants as our own GTEx-based analysis of the expression level impacts of protein-truncating variants that induce nonsense-mediated decay exhibited minimal tissue variability indicating that as long as the gene is expressed, transcriptome data can guide interpretation of multiple gene proximal variant effects (Teran et al. 2021).

For future rare disease studies, approaches that help identify the most informative cell types to study will also significantly aid in informed use of transcriptome data. We have recently seen the increased emergence of studies that demonstrate the utility of accessible cell types when the pathological tissue is hard to obtain. For example, Rentas et al. showed that lymphoblastoid cell lines derived from blood were highly relevant for neurodevelopmental rare diseases exhibiting broad isoform sharing with brain tissues and an overall ability to survey over 1700 rare neurodevelopmental disease genes (Rentas et al. 2020). Complementing this are new tools that use phenotype information to guide the tissue selection decision making process. Velluva et al. recently reported the Phenotype-Tissue Expression and Exploration (PTEE) tool to 
guide selection of analysis tissues in different disease contexts (Velluva et al. 2021). Future approaches may also begin to triangulate a subset of assayable cell types that are most relevant for a patient instead of selecting a single tissue to analyze. In our own work, we observed that combining undifferentiated induced pluripotent stem cells (iPSC) and blood transcriptomes from the same patient could enhance detection of outlier events and rapidly narrow candidate disease genes (Bonder et al. 2021). Likewise, Murdock et al. studied outliers in both blood and fibroblasts from the same patients (Murdock et al. 2021).

Beyond bulk transcriptomes, single cell multi-omics offers multiple emerging opportunities to map developmental and cell type specific effects. Large-scale single cell catalogs such as the Human Cell Atlas and Tabula Sapiens provide opportunities to map expression levels of candidate genes in highly-specific cell types across the human body (Regev et al. 2017; The Tabula Sapiens Consortium and Quake 2021). Future bulk and singlecell datasets from the Developmental GTEx (dGTEx) project will increase our understanding of human population variability in gene expression and splicing through development. Further, reference single-cell chromatin accessibility maps provide annotation of development and cell type-specific regulatory elements as potential targets of rare variant effects. The utility of these data is apparent in a recent map of single-cell chromatin accessibility in the developing cerebral cortex that identified an enrichment of de novo mutations from autism cases in the accessible chromatin of developing radial glial cells; an enrichment that authors noted was comparable to deleterious protein-coding mutations (Trevino et al. 2021). These reference single-cell data when combined with advances in non-coding variant effect prediction, will increasingly aid the prediction of development and cell-type specific pathological contexts and the experimental cellular proxies or analysis tissues for functional validation assays of patient-derived variants and mutations.

\section{COMPUTATIONAL METHODS FOR TRANSCRIPTOMICS IN RARE DISEASE}

Recent use of transcriptomics in rare diseases have led to multiple computational advances. One class of such advances have been pipelines and tools to define robust outlier gene expression or splicing events. Most prior studies have used a combination of defining Z-scores thresholds and/or assessed differential expression with DEseq2 (Love, Anders, and Huber 2014). This approach requires careful normalization of control data with respect to case data as there are often few rare disease samples sequenced separately from the majority of reference healthy controls. One approach to overcoming this has been to model and regress out known and latent factors across sequencing batches (Frésard et al. 2019). Separate computational tools called OUTRIDER and FRASER have been developed specifically for the task of rare disease diagnosis with RNA-seq by providing an end-to-end approach for correcting technical noise and providing a statistical test for expression and splicing outliers (Brechtmann et al. 2018; Mertes et al. 2021). These tools model latent factors using an autoencoder and report statistical significance from a negative-binomial or beta-binomial distribution. For splicing alone, the LeafCutterMD tool has provided an approach to detecting outliers and was designed to overcome class imbalance issues present when comparing small numbers of patient samples to multiple controls (Jenkinson et al. 2020). For each outlier detection approach, once a patient's transcriptome sample has been processed, there remains the possibility that too many outliers are detected due to sample specific issues that corrupt the measurement of many genes. To 
address this, we have often removed or reprocessed samples with abundant outliers (i.e., more than 50). Additionally, defining a meaningful outlier threshold for investigation can vary across studies and samples. In the cases where there is a single known candidate gene, the threshold for outlier effects may benefit from being reduced or in some cases manually inspected (Lee et al. 2020).

Another computational approach for guiding identification of causal genes and variants in rare disease transcriptomes has relied on allele-specific-expression (ASE) analysis. ASE is measurable by assessing the relative abundance of RNA sequencing read ratios over heterozygous coding variant sites; however, such variants need not be causal themselves (Castel et al. 2015). Several previously mentioned transcriptome studies from rare disease cohorts have used ASE as an additional signal to inform outlier events or potential haploinsufficiency. An additional study has also demonstrated more generally how ASE can identify likely Mendelian disease genes where protein-truncating variants escape nonsensemediated decay(Coban-Akdemir et al. 2018). To calculate ASE, most studies have used the WASP pipeline which aims to overcome mapping biases for non-reference allele-containing sequencing reads (van de Geijn et al. 2015). For rare disease transcriptome analysis, the computational tool ANEVA-DOT has further provided an approach to identify outlier ASE (Mohammadi et al. 2019). When applied to cases from Cummings et al., 76\% of cases had outlier ASE in a confirmed disease gene. Notably, this was among a small number of ASE outlier events detected per individual. Future computational approaches can likely increase the use of ASE in rare disease settings by integrating outlier expression and allele-specific expression into a combined outlier detection model.

Supplementing exome data, methods for calling variants from transcriptome data provides an opportunity for identifying rare coding alleles, splice sites and structural variants. One such tool, MINTIE, was applied to data from Cummings et al. and showed detection of 9 of 13 novel splice variants from ten individuals and the identification of a previously unobserved fusion product in the DMD gene (Cmero et al. 2021). Both Gonorazky and Yépez et al. further have applied GATK-based RNA-seq variant calling in their rare disease cohorts to extend coverage and identification of pathogenic variants into UTRs (Yépez et al. 2021; Gonorazky et al. 2019; "RNAseq Short Variant Discovery (SNPs + Indels)").

Integrative computational approaches that combine a patient's genome and transcriptome also provide exciting promise for aiding rare disease studies. This remains an area of nascent activity and most rare disease transcriptomics studies often assess candidate variants near outliers manually. To improve this, the computational tools RIVER and WATERSHED jointly model transcriptome outliers and genomic annotation of proximal variants to prioritize likely causal rare variants (Li et al. 2017; Ferraro et al. 2020). WATERSHED provides the opportunity to jointly assess gene expression, splicing and allele-specific expression outliers. These methods when combined with additional patient multi-omics are likely to continue to enhance prioritization of impactful rare variants. A key to this ongoing method development will be data sharing of whole genome, multi-omics and phenotype information from rare disease patients.

\section{ELIMINATING BARRIERS TO TRANSCRIPTOMICS IN RARE DISEASE STUDY AND DIAGNOSIS.}


Experimental advances over the last decade suggest that we are approaching a transition point where diagnostic use of transcriptomes may overcome current limitations related to developmental and cell type specificity. Already skin-biopsy derived fibroblasts and peripheral blood derived and transformed lymphoblastoid cell lines are used as source materials in rare disease transcriptomic studies. Gonorazky et al. took this one step further by transdifferentiating fibroblasts to myotubes and generating RNA-seq to study the diagnostic yield for patients with undiagnosed neuromuscular disorders (Gonorazky et al. 2019). Undifferentiated iPSC transcriptomes have demonstrated potential use for rare disease diagnoses by identifying disease-relevant outliers in a heterogeneous cohort of 65 rare disease patients (Bonder et al. 2021). As additional transdifferentiation and differentiation protocols become widely available and reproducible, the generation of cell-types of interest for transcriptomics can allow interrogation of a larger portion of the 'whole human transcriptome'. Just as genome sequencing has provided incremental diagnostic power versus exome sequencing, we believe interrogation of multiple cell-types through transcriptomic profiling may be an effective approach to enhancing diagnostic yield in rare disease. Further, the rapidly emerging availability of organoid systems and embryoid bodies to explore a range of developmental and cell type contexts expands the opportunities to study outlier effects in patients (Rhodes et al. 2022).

The availability of these cellular models is also providing new opportunities to rapidly test the impacts of genomic variants in previously hard to access contexts. Use of massively parallel reporter assays (MPRAs) have enabled testing thousands of variants for regulatory function in multiple primary cell types (White 2015). Splicing and 3'UTR variant MPRAs further provide opportunities to explore new classes of variant effects (Rosenberg et al. 2015; Griesemer et al. 2021). In parallel, high-throughput and multiplex CRISPRi and CRISPRa assays also provide the opportunity to inhibit and activate key regulatory regions harboring candidate variants and further provide new therapeutic opportunities to overcome haploinsufficiency in specific developmental and cell type contexts (Matharu et al. 2019). Strategies to test variant effects on gene expression and splicing in a range of different developmental and cell type contexts are already here.

With these advances it becomes possible to envision specific scenarios where a patient's phenotype with, or even without, their genotype can indicate a range of cellular contexts to study and that the presence of an aberrant molecular event will be sufficient to nominate a causal gene. In some cases, knowing the specific causal variant may be secondary to a diagnosis as the nature of an aberrant molecular event that integrates both the unseen rare variant and a patient's genetic background may be sufficient to support a diagnosis. There will of course be limitations as pathogenic variant effects that do not manifest on gene expression or downstream gene regulatory networks in any cellular context can be missed. Continued progress towards unlocking transcriptomics as a primary diagnostic tool at scale will require comprehensive maps of human cell types and their regulatory regions, activities already well underway. It will also require rapid and cost-effective, multiplexed cell culture, genetic or chemical perturbation assays that re-expose variant effects. Finally, it will require data sharing of reference population-scale databases to identify the normal ranges of molecular activity. With these resources, the future may see patients' care informed by knowledge of their genetic variants and additionally their personal transcriptomic profile. 


\section{Acknowledgments}

S.B.M., J.A.B. and M.T.W. are supported by NIH grants U01HG010218 (UDN) and U01HG011762 (GREGoR). S.B.M. is a consultant for BioMarin Pharmaceutical, Myome Inc. and Tenaya Therapeutics.

\section{References}

100,000 Genomes Project Pilot Investigators, Damian Smedley, Katherine R. Smith, Antonio Martin, Ellen A. Thomas, Ellen M. McDonagh, Valentina Cipriani, et al. 2021. "100,000 Genomes Pilot on Rare-Disease Diagnosis in Health Care - Preliminary Report." The New England Journal of Medicine 385 (20): 1868-80.

Ankala, Arunkanth, Cristina da Silva, Francesca Gualandi, Alessandra Ferlini, Lora J. H. Bean, Christin Collins, Alice K. Tanner, and Madhuri R. Hegde. 2015. "A Comprehensive Genomic Approach for Neuromuscular Diseases Gives a High Diagnostic Yield." Annals of Neurology 77 (2): 206-14.

Ayturk, Ugur M., Javier A. Couto, Steven Hann, John B. Mulliken, Kaitlin L. Williams, August Yue Huang, Steven J. Fishman, et al. 2016. "Somatic Activating Mutations in GNAQ and GNA11 Are Associated with Congenital Hemangioma." American Journal of Human Genetics 98 (6): 1271.

Baxter, Samantha M., Jennifer E. Posey, Nicole J. Lake, Nara Sobreira, Jessica X. Chong, Steven Buyske, Elizabeth E. Blue, et al. Feb, 9 2022. "Centers for Mendelian Genomics: A Decade of Facilitating Gene Discovery." Genetics in Medicine. https://doi.org/10.1016/j.gim.2021.12.005.

Bomba, Lorenzo, Klaudia Walter, and Nicole Soranzo. 2017. "The Impact of Rare and LowFrequency Genetic Variants in Common Disease." Genome Biology 18 (1): 77.

Bonder, Marc Jan, HipSci Consortium, Craig Smail, Michael J. Gloudemans, Laure Frésard, David Jakubosky, Matteo D'Antonio, et al. 2021. "Identification of Rare and Common Regulatory Variants in Pluripotent Cells Using Population-Scale Transcriptomics." Nature Genetics. https://doi.org/10.1038/s41588-021-00800-7.

Brechtmann, Felix, Christian Mertes, Agnè Matusevičiūtè, Vicente A. Yépez, Žiga Avsec, Maximilian Herzog, Daniel M. Bader, Holger Prokisch, and Julien Gagneur. 2018.

"OUTRIDER: A Statistical Method for Detecting Aberrantly Expressed Genes in RNA Sequencing Data." American Journal of Human Genetics 103 (6): 907-17.

Castel, Stephane E., Ami Levy-Moonshine, Pejman Mohammadi, Eric Banks, and Tuuli Lappalainen. 2015. "Tools and Best Practices for Data Processing in Allelic Expression Analysis." Genome Biology. https://doi.org/10.1186/s13059-015-0762-6.

Claringbould, Annique, and Judith B. Zaugg. 2021. "Enhancers in Disease: Molecular Basis and Emerging Treatment Strategies." Trends in Molecular Medicine 27 (11): 1060-73.

Clark, Michelle M., Zornitza Stark, Lauge Farnaes, Tiong Y. Tan, Susan M. White, David Dimmock, and Stephen F. Kingsmore. 2018. "Meta-Analysis of the Diagnostic and Clinical Utility of Genome and Exome Sequencing and Chromosomal Microarray in Children with Suspected Genetic Diseases." NPJ Genomic Medicine 3 (1): 1-10.

Cmero, Marek, Breon Schmidt, lan J. Majewski, Paul G. Ekert, Alicia Oshlack, and Nadia M. Davidson. 2021. "MINTIE: Identifying Novel Structural and Splice Variants in Transcriptomes Using RNA-Seq Data." Genome Biology 22 (1): 296.

Coban-Akdemir, Zeynep, Janson J. White, Xiaofei Song, Shalini N. Jhangiani, Jawid M. Fatih, Tomasz Gambin, Yavuz Bayram, et al. 2018. "Identifying Genes Whose Mutant Transcripts Cause Dominant Disease Traits by Potential Gain-of-Function Alleles." American Journal of Human Genetics 103 (2): 171-87.

Cummings, Beryl B., Jamie L. Marshall, Taru Tukiainen, Monkol Lek, Sandra Donkervoort, A. 
Reghan Foley, Veronique Bolduc, et al. 2017. "Improving Genetic Diagnosis in Mendelian Disease with Transcriptome Sequencing." Science Translational Medicine 9 (386).

https://doi.org/10.1126/scitranslmed.aal5209.

Deciphering Developmental Disorders Study. 2017. "Prevalence and Architecture of de Novo Mutations in Developmental Disorders." Nature 542 (7642): 433-38.

Ergin, Selvi, Nasim Kherad, and Meryem Alagoz. 2022. "RNA Sequencing and Its Applications in Cancer and Rare Diseases." Molecular Biology Reports, January. https://doi.org/10.1007/s11033-021-06963-0.

Ferraro, Nicole M., Benjamin J. Strober, Jonah Einson, Nathan S. Abell, Francois Aguet, Alvaro N. Barbeira, Margot Brandt, et al. 2020. "Transcriptomic Signatures across Human Tissues Identify Functional Rare Genetic Variation.” Science 369 (6509). https://doi.org/10.1126/science.aaz5900.

Frésard, Laure, Craig Smail, Nicole M. Ferraro, Nicole A. Teran, Xin Li, Kevin S. Smith, Devon Bonner, et al. 2019. "Identification of Rare-Disease Genes Using Blood Transcriptome Sequencing and Large Control Cohorts." Nature Medicine 25 (6): 911-19.

Geijn, Bryce van de, Graham McVicker, Yoav Gilad, and Jonathan K. Pritchard. 2015. "WASP: Allele-Specific Software for Robust Molecular Quantitative Trait Locus Discovery." Nature Methods 12 (11): 1061-63.

Gonorazky, Hernan D., Sergey Naumenko, Arun K. Ramani, Viswateja Nelakuditi, Pouria Mashouri, Peiqui Wang, Dennis Kao, et al. 2019. "Expanding the Boundaries of RNA Sequencing as a Diagnostic Tool for Rare Mendelian Disease." American Journal of Human Genetics 104 (5): 1007.

Griesemer, Dustin, James R. Xue, Steven K. Reilly, Jacob C. Ulirsch, Kalki Kukreja, Joe R. Davis, Masahiro Kanai, et al. 2021. "Genome-Wide Functional Screen of 3'UTR Variants Uncovers Causal Variants for Human Disease and Evolution." Cell 184 (20): 5247-60.e19.

Iglesias, Alejandro, Kwame Anyane-Yeboa, Julia Wynn, Ashley Wilson, Megan Truitt Cho, Edwin Guzman, Rebecca Sisson, Claire Egan, and Wendy K. Chung. 2014. "The Usefulness of Whole-Exome Sequencing in Routine Clinical Practice." Genetics in Medicine: Official Journal of the American College of Medical Genetics 16 (12): 922-31.

Jacob, Howard J., Kelly Abrams, David P. Bick, Kent Brodie, David P. Dimmock, Michael Farrell, Jennifer Geurts, et al. 2013. "Genomics in Clinical Practice: Lessons from the Front Lines." Science Translational Medicine 5 (194): 194cm5.

Jenkinson, Garrett, Yang I. Li, Shubham Basu, Margot A. Cousin, Gavin R. Oliver, and Eric W. Klee. 2020. "LeafCutterMD: An Algorithm for Outlier Splicing Detection in Rare Diseases." Bioinformatics 36 (17): 4609-15.

Keinan, Alon, and Andrew G. Clark. 2012. "Recent Explosive Human Population Growth Has Resulted in an Excess of Rare Genetic Variants." Science 336 (6082): 740-43.

Kopajtich, Robert, Dmitrii Smirnov, Sarah L. Stenton, Stefan Loipfinger, Chen Meng, Ines F. Scheller, Peter Freisinger, et al. 2021. "Integration of Proteomics with Genomics and Transcriptomics Increases the Diagnostic Rate of Mendelian Disorders." bioRxiv. medRxiv. https://doi.org/10.1101/2021.03.09.21253187.

Kremer, Laura S., Daniel M. Bader, Christian Mertes, Robert Kopajtich, Garwin Pichler, Arcangela luso, Tobias B. Haack, et al. 2017. "Genetic Diagnosis of Mendelian Disorders via RNA Sequencing." Nature Communications 8 (June): 15824.

Lee, Hane, Joshua L. Deignan, Naghmeh Dorrani, Samuel P. Strom, Sibel Kantarci, Fabiola Quintero-Rivera, Kingshuk Das, et al. 2014. "Clinical Exome Sequencing for Genetic Identification of Rare Mendelian Disorders." JAMA: The Journal of the American Medical Association 312 (18): 1880-87.

Lee, Hane, Alden Y. Huang, Lee-Kai Wang, Amanda J. Yoon, Genecee Renteria, Ascia Eskin, Rebecca H. Signer, et al. 2020. "Diagnostic Utility of Transcriptome Sequencing for Rare Mendelian Diseases." Genetics in Medicine: Official Journal of the American College of 
Medical Genetics 22 (3): 490-99.

Li, Xin, GTEx Consortium, Yungil Kim, Emily K. Tsang, Joe R. Davis, Farhan N. Damani, Colby Chiang, et al. 2017. "The Impact of Rare Variation on Gene Expression across Tissues." Nature. https://doi.org/10.1038/nature24267.

Lord, Jenny, and Diana Baralle. 2021. "Splicing in the Diagnosis of Rare Disease: Advances and Challenges." Frontiers in Genetics 12 (July): 689892.

Love, Michael, Simon Anders, and Wolfgang Huber. 2014. "Differential Analysis of Count Data-the DESeq2 Package." Genome Biology 15 (550): 10-1186.

Macken, William L., Jana Vandrovcova, Michael G. Hanna, and Robert D. S. Pitceathly. 2021. "Applying Genomic and Transcriptomic Advances to Mitochondrial Medicine." Nature Reviews. Neurology 17 (4): 215-30.

Matharu, Navneet, Sawitree Rattanasopha, Serena Tamura, Lenka Maliskova, Yi Wang, Adelaide Bernard, Aaron Hardin, Walter L. Eckalbar, Christian Vaisse, and Nadav Ahituv. 2019. "CRISPR-Mediated Activation of a Promoter or Enhancer Rescues Obesity Caused by Haploinsufficiency." Science 363 (6424). https://doi.org/10.1126/science.aau0629.

Mertes, Christian, Ines F. Scheller, Vicente A. Yépez, Muhammed H. Çelik, Yingjiqiong Liang, Laura S. Kremer, Mirjana Gusic, Holger Prokisch, and Julien Gagneur. 2021. "Detection of Aberrant Splicing Events in RNA-Seq Data Using FRASER." Nature Communications 12 (1): 529.

Mohammadi, Pejman, Stephane E. Castel, Beryl B. Cummings, Jonah Einson, Christina Sousa, Paul Hoffman, Sandra Donkervoort, et al. 2019. "Genetic Regulatory Variation in Populations Informs Transcriptome Analysis in Rare Disease." Science 366 (6463): 35156.

Montgomery, Stephen B., Tuuli Lappalainen, Maria Gutierrez-Arcelus, and Emmanouil T. Dermitzakis. 2011. "Rare and Common Regulatory Variation in Population-Scale Sequenced Human Genomes." PLoS Genetics 7 (7): e1002144.

Murdock, David R., Hongzheng Dai, Lindsay C. Burrage, Jill A. Rosenfeld, Shamika Ketkar, Michaela F. Müller, Vicente A. Yépez, et al. 2021. "Transcriptome-Directed Analysis for Mendelian Disease Diagnosis Overcomes Limitations of Conventional Genomic Testing." The Journal of Clinical Investigation 131 (1). https://doi.org/10.1172/JCI141500.

NICUSeq Study Group, lan D. Krantz, Livija Medne, Jamila M. Weatherly, K. Taylor Wild, Sawona Biswas, Batsal Devkota, et al. 2021. "Effect of Whole-Genome Sequencing on the Clinical Management of Acutely III Infants With Suspected Genetic Disease: A Randomized Clinical Trial." JAMA Pediatrics 175 (12): 1218-26.

Posey, Jennifer E., Jill A. Rosenfeld, Regis A. James, Matthew Bainbridge, Zhiyv Niu, Xia Wang, Shweta Dhar, et al. 2016. "Molecular Diagnostic Experience of Whole-Exome Sequencing in Adult Patients." Genetics in Medicine: Official Journal of the American College of Medical Genetics 18 (7): 678-85.

Regev, Aviv, Sarah A. Teichmann, Eric S. Lander, Ido Amit, Christophe Benoist, Ewan Birney, Bernd Bodenmiller, et al. 2017. "The Human Cell Atlas." eLife 6 (December). https://doi.org/10.7554/elife.27041.

Rentas, Stefan, Komal S. Rathi, Maninder Kaur, Pichai Raman, lan D. Krantz, Mahdi Sarmady, and Ahmad Abou Tayoun. 2020. "Diagnosing Cornelia de Lange Syndrome and Related Neurodevelopmental Disorders Using RNA Sequencing." Genetics in Medicine: Official Journal of the American College of Medical Genetics 22 (5): 927-36.

Rhodes, Katherine, Kenneth A. Barr, Joshua M. Popp, Benjamin J. Strober, Alexis Battle, and Yoav Gilad. 2022. "Human Embryoid Bodies as a Novel System for Genomic Studies of Functionally Diverse Cell Types." eLife 11 (February). https://doi.org/10.7554/eLife.71361.

"RNAseq Short Variant Discovery (SNPs + Indels)." n.d. GATK. Accessed February 10, 2022. https://gatk.broadinstitute.org/hc/en-us/articles/360035531192?id=3891.

Rosenberg, Alexander B., Rupali P. Patwardhan, Jay Shendure, and Georg Seelig. 2015. 
"Learning the Sequence Determinants of Alternative Splicing from Millions of Random Sequences." Cell 163 (3): 698-711.

Saeidian, Amir Hossein, Leila Youssefian, Hassan Vahidnezhad, and Jouni Uitto. 2020.

"Research Techniques Made Simple: Whole-Transcriptome Sequencing by RNA-Seq for Diagnosis of Monogenic Disorders." The Journal of Investigative Dermatology 140 (6): 1117-26.e1.

Shashi, Vandana, Allyn McConkie-Rosell, Bruce Rosell, Kelly Schoch, Kasturi Vellore, Marie McDonald, Yong-Hui Jiang, Pingxing Xie, Anna Need, and David B. Goldstein. 2014. "The Utility of the Traditional Medical Genetics Diagnostic Evaluation in the Context of nextGeneration Sequencing for Undiagnosed Genetic Disorders." Genetics in Medicine: Official Journal of the American College of Medical Genetics 16 (2): 176-82.

Splinter, Kimberly, David R. Adams, Carlos A. Bacino, Hugo J. Bellen, Jonathan A. Bernstein, Alys M. Cheatle-Jarvela, Christine M. Eng, et al. 2018. "Effect of Genetic Diagnosis on Patients with Previously Undiagnosed Disease." The New England Journal of Medicine 379 (22): 2131-39.

Tan, Tiong Yang, Oliver James Dillon, Zornitza Stark, Deborah Schofield, Khurshid Alam, Rupendra Shrestha, Belinda Chong, et al. 2017. "Diagnostic Impact and Cost-Effectiveness of Whole-Exome Sequencing for Ambulant Children With Suspected Monogenic Conditions." JAMA Pediatrics 171 (9): 855-62.

Teran, Nicole A., Daniel C. Nachun, Tiffany Eulalio, Nicole M. Ferraro, Craig Smail, Manuel A. Rivas, and Stephen B. Montgomery. 2021. "Nonsense-Mediated Decay Is Highly Stable across Individuals and Tissues." American Journal of Human Genetics 108 (8): 1401-8.

The GTEx Consortium. 2020. "The GTEx Consortium Atlas of Genetic Regulatory Effects across Human Tissues." Science, September. https://science.sciencemag.org/content/369/6509/1318.abstract.

The Tabula Sapiens Consortium, and Stephen R. Quake. 2021. "The Tabula Sapiens: A Single Cell Transcriptomic Atlas of Multiple Organs from Individual Human Donors." bioRxiv. https://doi.org/10.1101/2021.07.19.452956.

Trevino, Alexandro E., Fabian Müller, Jimena Andersen, Laksshman Sundaram, Arwa Kathiria, Anna Shcherbina, Kyle Farh, et al. 2021. "Chromatin and Gene-Regulatory Dynamics of the Developing Human Cerebral Cortex at Single-Cell Resolution." Cell 184 (19): 5053-69.e23.

Velluva, Akhil, Maximillian Radtke, Susanne Horn, Bernt Popp, Konrad Platzer, Erind Gjermeni, Chen-Ching Lin, et al. 2021. "Phenotype-Tissue Expression and Exploration (PTEE) Resource Facilitates the Choice of Tissue for RNA-Seq-Based Clinical Genetics Studies." BMC Genomics 22 (1): 802.

White, Michael A. 2015. "Understanding How Cis-Regulatory Function Is Encoded in DNA Sequence Using Massively Parallel Reporter Assays and Designed Sequences." Genomics 106 (3): 165-70.

Wortmann, Saskia B., David A. Koolen, Jan A. Smeitink, Lambert van den Heuvel, and Richard J. Rodenburg. 2015. "Whole Exome Sequencing of Suspected Mitochondrial Patients in Clinical Practice." Journal of Inherited Metabolic Disease 38 (3): 437-43.

Yang, Yaping, Donna M. Muzny, Jeffrey G. Reid, Matthew N. Bainbridge, Alecia Willis, Patricia A. Ward, Alicia Braxton, et al. 2013. "Clinical Whole-Exome Sequencing for the Diagnosis of Mendelian Disorders." The New England Journal of Medicine 369 (16): 1502-11.

Yang, Yaping, Donna M. Muzny, Fan Xia, Zhiyv Niu, Richard Person, Yan Ding, Patricia Ward, et al. 2014. "Molecular Findings among Patients Referred for Clinical Whole-Exome Sequencing." JAMA: The Journal of the American Medical Association 312 (18): 1870-79.

Yépez, Vicente A., Mirjana Gusic, Robert Kopajtich, Christian Mertes, Nicholas H. Smith, Charlotte L. Alston, Rui Ban, et al. 2021. "Clinical Implementation of RNA Sequencing for Mendelian Disease Diagnostics." bioRxiv. medRxiv. https://doi.org/10.1101/2021.04.01.21254633. 
Yuen, Ryan K. C., Bhooma Thiruvahindrapuram, Daniele Merico, Susan Walker, Kristiina Tammimies, Ny Hoang, Christina Chrysler, et al. 2015. "Whole-Genome Sequencing of Quartet Families with Autism Spectrum Disorder." Nature Medicine 21 (2): 185-91.

Zeng, Yong, Gang Wang, Ence Yang, Guoli Ji, Candice L. Brinkmeyer-Langford, and James J. Cai. 2015. "Aberrant Gene Expression in Humans." PLoS Genetics 11 (1): e1004942.

Zhao, Jing, Idowu Akinsanmi, Dalia Arafat, T. J. Cradick, Ciaran M. Lee, Samridhi Banskota, Urko M. Marigorta, Gang Bao, and Greg Gibson. 2016. "A Burden of Rare Variants Associated with Extremes of Gene Expression in Human Peripheral Blood." American Journal of Human Genetics 98 (2): 299-309. 


\section{COLD SPRING HARBOR Molecular Case Studies}

\section{TOWARDS TRANSCRIPTOMICS AS A PRIMARY TOOL FOR RARE DISEASE INVESTIGATION}

Stephen B Montgomery, Jonathan A Bernstein and Matthew T Wheeler

Cold Spring Harb Mol Case Stud published online February 25, 2022

Access the most recent version at doi: $10.1101 / \mathrm{mcs} . \mathrm{a} 006198$

Published online February 25, 2022 in advance of the full issue.

Accepted Peer-reviewed and accepted for publication but not copyedited or typeset; accepted

Manuscript manuscript is likely to differ from the final, published version. Published onlineFebruary 25,2022 in advance of the full issue.

Creative This article is distributed under the terms of the

Commons http://creativecommons.org/licenses/by/4.0/, which permits unrestricted reuse and

License redistribution provided that the original author and source are credited.

Email Alerting Receive free email alerts when new articles cite this article - sign up in the box at the Service top right corner of the article or click here. 